\title{
A broadband millimeter-wave spectrometer Z-spec: sensitivity and ULIRGs
}

Hanae Inami, Matt Bradford, James Aguirre, Lieko Earle, Bret Naylor, et al.

Hanae Inami, Matt Bradford, James Aguirre, Lieko Earle, Bret Naylor, Hideo Matsuhara, Jason Glenn, Hien Nguyen, James J. Bock, Jonas Zmuidzinas, Youichi Ohyama, "A broadband millimeter-wave spectrometer Zspec: sensitivity and ULIRGs," Proc. SPIE 7020, Millimeter and Submillimeter Detectors and Instrumentation for Astronomy IV, 70201T (19 July 2008); doi: 10.1117/12.788711

Event: SPIE Astronomical Telescopes + Instrumentation, 2008, Marseille, France 


\title{
A broadband millimeter-wave spectrometer-Z-spec: Sensitivity and ULIRGs
}

\author{
Hanae Inami* ${ }^{\mathrm{a}, \mathrm{b}}$, Matt Bradford ${ }^{\mathrm{c}, \mathrm{d}}$, James Aguirre ${ }^{\mathrm{e}}$, Lieko Earle ${ }^{\mathrm{e}}$, Bret Naylor ${ }^{\mathrm{d}}$, Hideo Matsuhara ${ }^{\mathrm{b}}$, \\ Jason Glenn ${ }^{\mathrm{e}}$, Hien Nguyen ${ }^{\mathrm{d}}$, James J. Bock ${ }^{\mathrm{c}, \mathrm{d}}$, Jonas Zmuidzinas ${ }^{\mathrm{e}}$, Youichi Ohyama ${ }^{\mathrm{f}}$ \\ ${ }^{a}$ The Graduate Univ. for Advanced Studies, 3-1-1 Yoshinodai, Sagamihara, Kanagawa 229-8510 \\ Japan \\ ${ }^{\mathrm{b}}$ Institute of Space and Astronautical Science, Japan Aerospace Exploration Agency, 3-1-1 \\ Yoshinodai, Sagamihara, Kanagawa 229-8510 Japan \\ ${ }^{\mathrm{c}}$ California Institute of Technology, 1200 E. California Blvd., Pasadena CA 91125 \\ d Jet Propulsion Lab., 4800 Oak Grove Drive, Pasadena, CA 91109 \\ ${ }^{\mathrm{e}}$ Univ. of Colorado at Boulder, CASA / ARL 1255 38th St. Boulder, CO 80303 \\ ${ }^{\mathrm{f}}$ Institute of Astronomy and Astrophysics, Academia Sinica, P.O. Box 23-141, Taipei 10617, \\ Taiwan, R.O.C.
}

\begin{abstract}
Z-Spec ${ }^{[1,2,3,4,5,6]}$ is a cryogenic, broadband, millimeter-wave grating spectrometer. It is capable of obtaining many spectral lines simultaneously because of its unprecedented broad bandwidth (185-305GHz). The bandpass covers the $1 \mathrm{~mm}$ atmospheric transmission window with a resolving power of 250-400. Z-Spec uses 160 silicon nitride micromesh bolometers cooled down to less than $100 \mathrm{mK}$ for background-limited performance. The unique capability of Z-Spec to detect multiple lines simultaneously allows us to obtain information efficiently on the physical and chemical conditions of nearby Ultra-luminous Infrared Galaxies (ULIRGs) powered by starbursts or Active Galactic Nuclei. Here we report on new millimeter-wave broadband data for ULIRGs acquired with Z-Spec and the noise performance and achieved sensitivity in observations with the CSO. We found that during the observations the noise scales with the atmospheric opacity and can be explained well by our sensitivity model, considering the photon noise originating from the sky and the telescope, as well as the detector and electronics noise. The photon noise is found to dominate the total noise.
\end{abstract}

Keywords: millimeter, spectrometer, line survey, sensitivity, ULIRGs

\section{INTRODUCTION}

We have operated Z-Spec at the Caltech Submillimeter Observatory (CSO) in Hawaii for routine observations from 2006. Z-Spec was originally designed for molecular line surveys of both high-z and local galaxies. It covers the entire 1 $\mathrm{mm}$ atmosphere transmission window $(185-305 \mathrm{GHz})$, dispersion is accomplished with a waveguide diffraction grating. This extremely broadband millimeter spectrometer allows us to measure redshifts of high-z submillimeter galaxies sources by using multiple carbon monoxide $\left({ }^{12} \mathrm{CO}\right)$ molecular lines, and also to undertake line surveys of nearby galaxies.

\subsection{Z-Spec design}

An ordinary three dimensional grating spectrometer requires several cubic meters of volume to achieve the same spectroscopic resolution as Z-Spec, but Z-Spec is quite unique in its compact design, as shown in Figure 1. Its grating elements are based on the Rowland grating ${ }^{[7]}$ and the waveguide is a parallel plate. Astronomical source radiation comes in from an input feedhorn and is dispersed by grating arc that has 480 facets with blaze angle of 27 degrees (the resolving power is $\sim 250-400$ ). The dispersed light is detected at the focal plane, which is composed with $160 \mathrm{Si}_{3} \mathrm{~N}_{4}$ micromesh bolometers.

*hanae@ir.isas.jaxa.jp; phone +81-42-751-8076; fax +81-42-786-7202

Millimeter and Submillimeter Detectors and Instrumentation for Astronomy IV edited by William D. Duncan, Wayne S. Holland, Stafford Withington, Jonas Zmuidzinas Proc. of SPIE Vol. 7020, 70201T, (2008) · 0277-786X/08/\$18 - doi: 10.1117/12.788711 


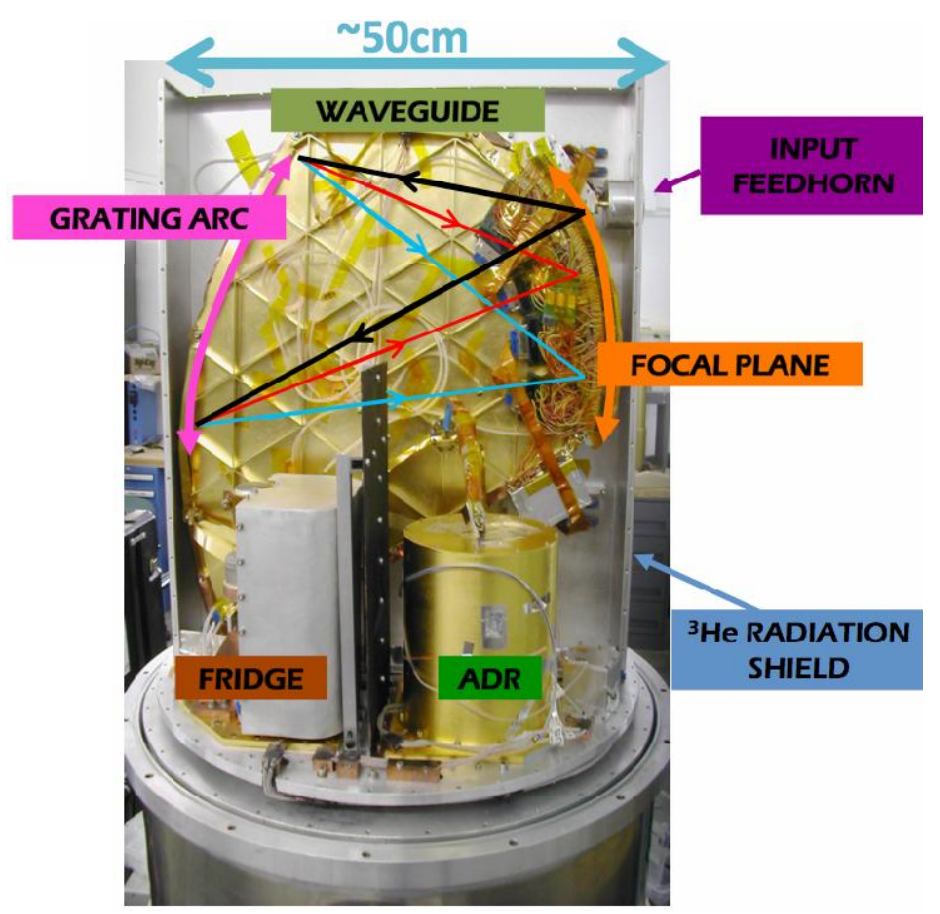

Fig. 1. The inside of the Z-Spec cold instrument. The size of the entire parallel waveguide module is $68 \mathrm{~cm} \times 48 \mathrm{~cm} \times$ $3.3 \mathrm{~cm}$. We use an ADR and a ${ }^{3} \mathrm{He} /{ }^{4} \mathrm{He}$ closed-cycle fridge, two stages, to cool the feedhorn, grating and detector array down to $\sim 60 \mathrm{mK}$. Black arrows in the pictures indicate incoming source light that will be dispersed (shown in arrows; the colors can be seen in electronic version as blue and red corresponding to shorter and longer wavelength).

The feedhorn, grating and detector array are cooled down to $60 \mathrm{mK}$ to acheive background limited performance. Two stages supply cooling: an adiabatic demagnetization refrigerator (ADR) and a ${ }^{3} \mathrm{He} /{ }^{4} \mathrm{He}$ closed-cycle fridge.

In Z-Spec's cold hardware, there are no moving parts. It is extremely compact compared to a conventional grating spectrograph, thus, it is also suitable for a focal-plane instrument onboard future space missions with cold telescopes; e.g. BLISS on SPICA ${ }^{[8]}$.

\subsection{Scientific motivations}

Z-Spec has two main scientific objectives. One is to determine the redshifts of high-z luminous dusty galaxies with line emissions from the CO ladder (Fig. 2) and the other is to obtain multiple molecular lines of nearby ULIRGs to probe their physical and chemical conditions.

\subsubsection{Measuring redshifts of high-z galaxies}

Z-Spec was designed for measurement of redshifts of high-z, luminous, dusty galaxies by simultaneously detecting two or more CO lines. Luminous dusty galaxies are often referred to as submillimeter galaxies, often they have extremely faint or nonexistent optical counterparts but are very luminous $\left(>10^{12} \mathrm{~L}_{\odot}\right)$ integrated over the far-infrared to millimeter wave range because they are heavily dust-enshrouded ${ }^{[8]}$. SCUBA ${ }^{[9]}$, MAMBO $^{[10]}$, Bolocam ${ }^{[11]}$, and AzTEC (on ASTE) have operated deep observations with their large bolometer array camera to find these types of galaxies. Multiband observations have suggested their powerful emission is due to thermal radiation from starbursts or/and active galactic nuclei (AGN). The galaxies possibly are progenitors of present-day elliptical galaxies but we need to determine what exactly occurs in the galaxies. Therefore, we need follow-up observations and spectroscopic data. Recently, many challenging investigations show successful measurements of redshift for a subsample of submillimeter galaxies with 


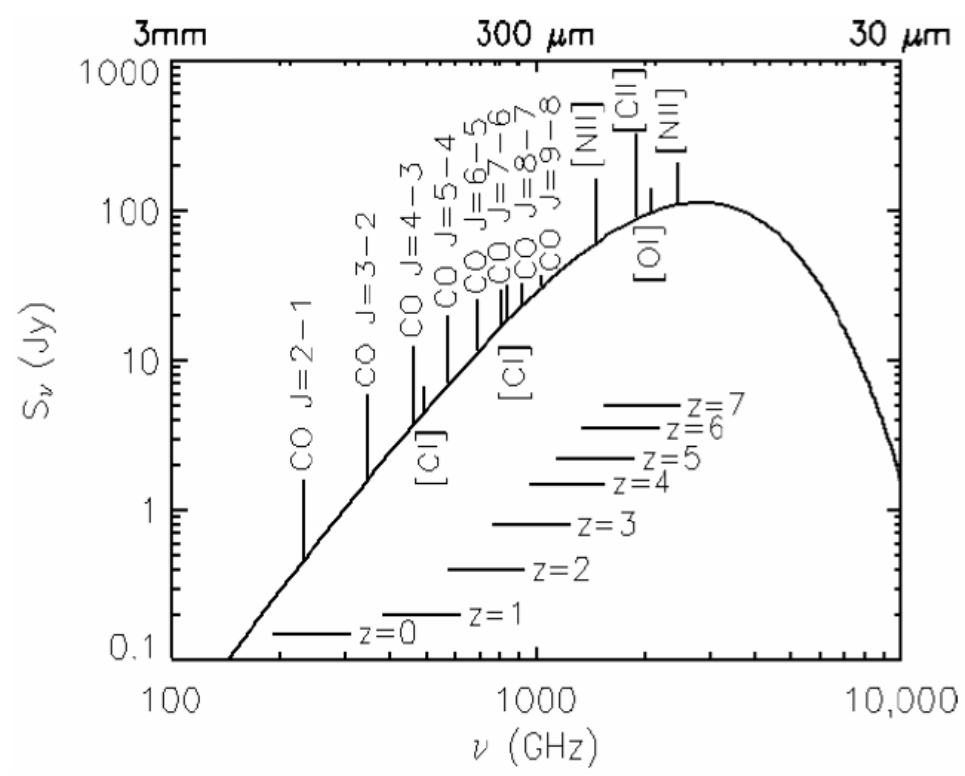

Fig. 2 Lines from the CO ladder and strong atomic/ionic fine structure lines overlaid on the Arp220 continuum which is modeled as $42 \mathrm{~K}$ gray-body spectrum (Scoville et al. 1991) ${ }^{[14]}$. The horizontal lines are the Z-Spec bandwidth for various redshifts indicated. If a source lies at $\mathrm{z}>0.9$, we can observe more than two CO lines in the bandwidth and we can obtain its redshift.

radio identifications. The as done with the Keck LRIS spectrograph, showing their radio-selected bright sample has a median redshift at $2.4^{[12]}$. One the other hand, Wang et al. (2006) found that faint far-infrared/submillimeter galaxies tend to lie at redshift less than one by using cross-correlation with near-infrared and Spitzer catalogs ${ }^{[13]}$. Observing many submillimeter galaxies with sensitive millimeter-wave spectrometers is important: it is difficult to obtain redshifts at optical and near infrared due to dust extinction, and the beam size of submillimeter is too large to identify the counterpart in the optical and near infrared images.

In order to overcome the problems, we have developed a spectrometer with very wide bandwidth covering whole 1 $\mathrm{mm}$ atmosphere transmission window to detect multiple $\mathrm{CO}$ rotational transition lines simultaneously. In case of the source at $z \geq 0.9$ (Fig. 1), we can have more than two ${ }^{12} \mathrm{CO}$ lines in the band and we can easily identify them because the ${ }^{12} \mathrm{CO}$ lines are likely the strongest in millimeter lines and they are regularly spaced in frequency. We are able to obtain the lines simultaneously and directly measure redshifts with the ratios of line frequencies.

\subsubsection{Nearby (U)LIRGs line surveys}

Luminous infrared galaxies (LIRGs) and ultra luminous infrared galaxies (ULIRGs) are the most infrared-luminous objects in the local universe, with $8-1000 \mu \mathrm{m}$ integrated luminosities $\mathrm{L}_{\mathrm{IR}}$ of $10^{11} \mathrm{~L}_{\odot}<\mathrm{L}_{\mathrm{IR}}<10^{12} \mathrm{~L}_{\odot}$ and $10^{12} \mathrm{~L}_{\odot}<\mathrm{L}_{\mathrm{IR}}<$ $10^{13} \mathrm{~L}_{\odot}$, respectively. These were first discovered in the $1980 \mathrm{~s}$ with $I R A S$. There has been a long-lasting debate about the energy source of these galaxies: mass accretion on to super massive black holes (Active Galactic Nuclei: AGN) or starbursts in the central regions obscured by dust ${ }^{[15,16]}$. Moreover, whatever the nature of the central energy sources, the input of such enormous amounts of energy and momentum into the surrounding interstellar matter has profound effects on the story of galaxy evolution. Therefore, unveiling the nature of local LIRGs and ULIRGs is a fundamental issue for understanding the energy production history of the universe.

Because the central regions of (U)LIRGs are surrounded by dust, observations at millimeter wavelengths are quite useful since they do not suffer from dust extinction. (U)LIRGs are known to emit strong CO lines as well as lines from many kinds of molecular species. Some of the lines trace dense gas which harbors star formation activity. Z-Spec has capability to obtain many molecular lines simultaneously for unbiased line surveys of nearby (U)LIRGs thanks to its large bandpass: ${ }^{12} \mathrm{CO}(\mathrm{J}=2 \rightarrow 1), \quad{ }^{13} \mathrm{CO}(\mathrm{J}=2 \rightarrow 1), \quad \mathrm{C}^{18} \mathrm{O}(\mathrm{J}=2 \rightarrow 1), \quad \mathrm{CN}(\mathrm{J}=2 \rightarrow 1), \quad \mathrm{HCN}(\mathrm{J}=3 \rightarrow 2), \quad \mathrm{HCO}^{+}(\mathrm{J}=3 \rightarrow 2)$, $\mathrm{HNC}(\mathrm{J}=3 \rightarrow 2)$ and $\mathrm{CS}(\mathrm{J}=5 \rightarrow 4,6 \rightarrow 5)$. 
Another interesting capability of Z-Spec is that we can obtain a broadband continuum from each target, in contrast to heterodyne spectrometers. In addition, Z-Spec is also capable to discover new molecular lines with line surveys. More details are given in section 3.

\section{OBSERVED SENSITIVITY AND FUNDAMENTAL LIMITS}

To understand the instrument performance, we compared the measured sensitivity with a model containing noise sources. Z-Spec uses low-temperature resistive bolometers. Absorbed energy from photons increases the absorber temperature which is sensed with a thermistor (temperature-dependent resistor). The resistance is measured via output
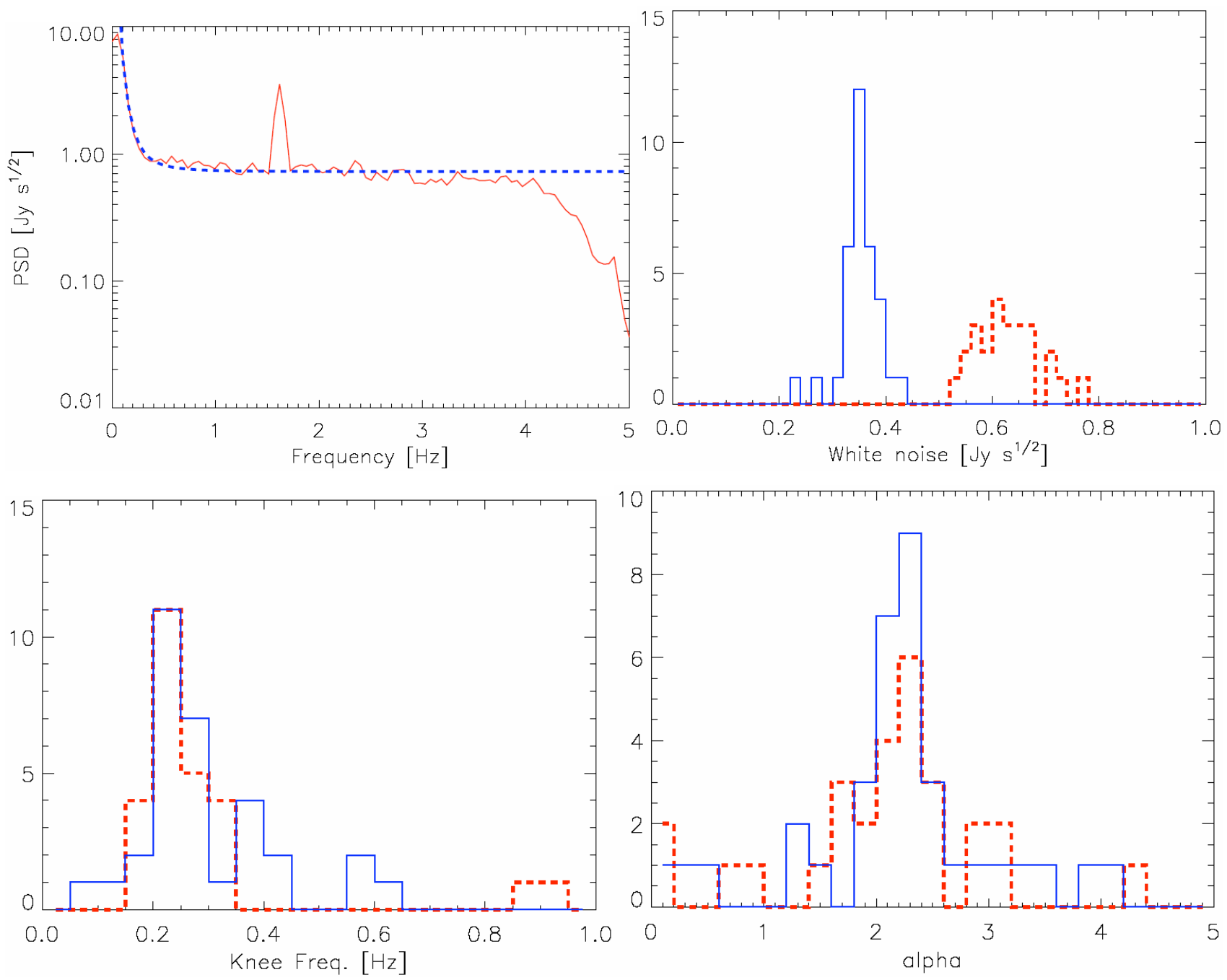

Fig. 3. [Upper left] PSD of the detector output voltage (channel No.92 at $240.9 \mathrm{GHz}$ ). The observation was made on April 16, $2008\left[\tau_{225}=0.18\right]$ ). The red solid line is the weighted average of PSDs obtained in observations of all nod cycles, the blue dashed line is the fit (Equation (1)) and the flat part corresponds to the white noise level. The rest of figures are histograms of the white noise level (upper right), knee frequency (bottom left), and the index alpha (bottom right) which are defined in Equations (1) and (2). Cmparison of the atmospheric optical depth at $225 \mathrm{GHz}\left(\tau_{225}\right)$ lower (blue solid line) than and higher (red dashed line) than 0.1. [Colors can be seen in the electric version] 
voltage, since the bolometer is operated with a constant current bias. In addition to the Poisson fluctuations in the photon stream itself, sources of noise also include the thermal transport in the bolometer, Johnson noise, and the noise of the JFET amplifiers. These noise sources are independent, and thus contribute to the total noise as a quadrature sum of each noise source. The photon noise is calculated below, while for the other terms, we adopt a constant noise value of $6.4 \times 10^{-18} \mathrm{~W} \mathrm{~Hz}^{-1 / 2}$, which approximately accounts for all of the non-photon noise sources - specifically, the noise from optical load inside the instrument $\left(\sim 4 \times 10^{-18} \mathrm{~W} \mathrm{~Hz}^{-1 / 2}\right)$, and the combination of thermal transport noise in the bolometers and the noise voltage of the JFET amplifiers (corresponding to $\sim 5 \times 10^{-18} \mathrm{~W} \mathrm{~Hz}^{-1 / 2}$ ).

\subsection{Observed sensitivity}

For each detector, the uncertainty in an observation is estimated by examining the power spectral density (PSD) of the bolometer voltage, as shown in Figure 3 upper left panel. The signal is at the chop frequency, and its uncertainty is estimated by averaging the PSD close to the chop frequency. The mean signal is then the weighted mean of the signal in all of the nods. To understand the noise properties, we fit the PSD to a two-component model:

$$
P S D=A\left(\frac{1}{f}\right)^{\alpha}+c .
$$

Here $c$ is $\mathrm{s}$ the white noise which should include all fundamental photon and thermal noise sources (those described above). The first term represents low-frequency noise, (called $1 / f$ noise), which could arise from a variety of sources, but is generally due to fluctuations in sky power for a millimeter-wave bolometer system. The knee frequency $f_{k}$ is defined as the frequency where the white noise is equal to the $1 / f$ noise,

$$
A\left(\frac{1}{f_{k}}\right)^{\alpha}=\text { white noise }
$$

and is a minimum frequency for useful observations. As Fig. 3 show in the upper left panel, Z-Spec's operating frequency is well above the knee frequency. Additionally, when $1 / f$ component increases, we can increase the chop frequency to stay above the knee frequency.

To show the range of values for these fit parameters, we have generated histograms, shown in Figure 3. These are expected depend on atmosphere opacity, therefore we divide our 2008 April observations with the atmospheric optical depth $\tau_{225}=0.1$ to compare the parameter behaviors between the high and low $\tau_{225}$. The result shows the white noise has a strong dependence on atmospheric opacity, but the $1 / f$ noise does not.

\subsection{Comparison with fundamental photon noise}

We use $\mathrm{Si}_{3} \mathrm{~N}_{4}$ micromesh bolometers which are semiconductor-based for Z-Spec. The noise equivalent power (NEP) is normally specified in units of $\mathrm{W} \mathrm{Hz}^{-1 / 2}$ and defined as the single power that gives an rms signal-to-noise ratio of unity in a system that has a bandpass of $1 \mathrm{~Hz}$. The NEP of a bolometer is related to the operating temperature $T$ and its thermal conductance $G$ by the formula $N E P_{\text {bolo }} \sim \sqrt{4 k T^{2} G}$, similar to the formula for Johnson noise in a resistor.

There are two kinds of noises which limit Z-Spec's performance, one can be controlled (e.g. bolometer noise), and the another cannot. The latter noise, known as the photon background noise, obeys the Poisson statistics. Understanding this intrinsic noise is important for Z-Spec sensitivity.

The dominant photon load on Z-Spec's detectors is due to the atmosphere and telescope background. We model this background as graybody radiation, which can be described with the Planck distribution of photon occupation number $n_{\text {phot }_{v}}(T)$. It presents the number of photons of a particular energy (or equivalently, frequency, $v$ ) and depends on the blackbody at temperature $T$ :

$$
n_{\text {phot }_{v}}(T)=£\left(\frac{1}{e^{h v / k T}-1}\right)
$$


where $\varepsilon$ is the emissivity of background radiation, $h$ is Planck's constant and $k$ is Boltzmann's constant. The NEP due to this photon distribution is given by

$$
N E P_{\text {phot }}=h v \sqrt{2 \Delta v n_{p h o t}\left(n_{p h o t}-1\right)}
$$

where $\Delta v$ is the detector bandwidth. We can rewrite the photon occupation number distribution by taking account of the effect of the optical efficiency of the instrument and the atmosphere transparency at the Z-Spec bandpass. The atmospheric opacity reduces the number of astrophysical phoons that get to the telescope and the less-than-unity optical efficiency of the instruments further reduces the number. Supposing atmosphere and telescope are the same temperature, the proper photon distribution $\bar{n}$ to use in the equation for photon NEP is

$$
\bar{n}_{p h o t}=\eta\left[1-\xi_{\text {atm }}\left(v: \tau_{225}\right)\right] n_{\text {phot }}
$$

where $\eta$ is the instrument optical efficiency including the detector absorption efficiency, $\xi$ is the forward efficiency of the telescope ( $\sim 0.9$ for the CSO) and $t_{\text {atm }}$ is the atmosphere transmission, which is a function of frequency and the observing conditions characterized by $\tau_{225}$, the atmospheric optical depth at $225 \mathrm{GHz}$. The total noise at the detector, $\mathrm{NEP}_{\text {tot, }}$ is gained by combining the detector and electronics noise in quadrature sum.

The noise equivalent flux density (NEFD) describes the sensitivity at the top of the atmosphere. The NEFD is a straightforward scaling of the $\mathrm{NEP}_{\text {tot }}$ given by

$$
N E P D=2 \times 2 \times \frac{1}{\eta \xi T_{a t m}} \times \frac{1}{v A_{t e l} 10^{-26}} \times \frac{N E P_{t o t}}{\sqrt{2}}
$$

The two initial factors of two come from chopping and polarization. Chopping increased the NEFD by two factors of $\sqrt{2}$ : one because half the integration time is spent looking off source and the other because two measurements with roughly equal noise are subtracted from each other. Since Z-Spec is a single polarization instrument and the polarization of the light from the galaxy is very small, we need twice as much power at the top of the atmosphere to generate a given signal at the detectors. The next set of factors come from the transmission efficiency of the instrument $(\eta)$, the telescope $\left(\zeta=\zeta_{0} \exp (4 \pi \sigma / \lambda)\right.$, the Ruze-corrected aperture efficiency) and the atmosphere $\left(T_{a t m}\right)$. The final set of factors converts from units of power to units of $\mathrm{Jy}\left(10^{-26} \mathrm{~W} \mathrm{~m}^{-2} \mathrm{~Hz}^{-1}\right)$, where $A_{\text {tel }}=\pi D^{2} / 4$, the area of the CSO $(\mathrm{D}=10.4 \mathrm{~m})$. The final $\sqrt{2}$
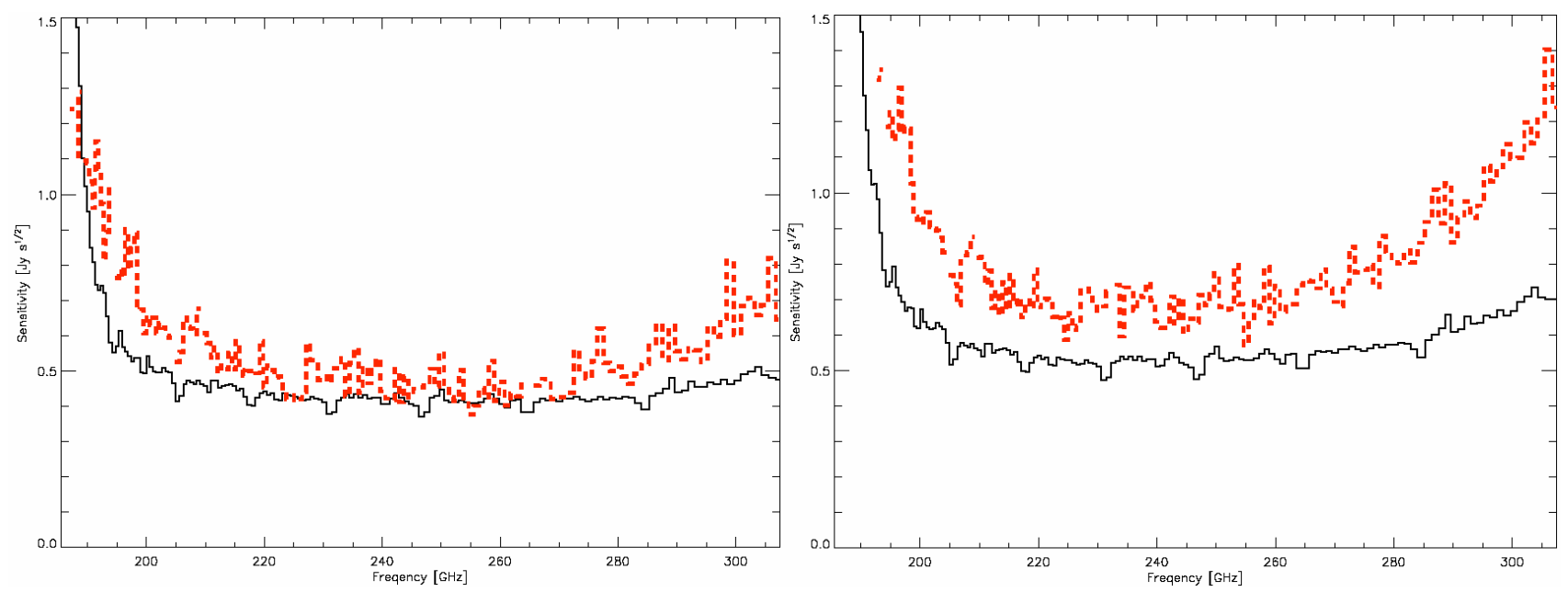

Fig. 4. Comparison of the sensitivities between those estimated from the white noise of the PSDs of the bolometer signals and the Z-Spec sensitivity model (see text). The black solid line is the model and the red dashed line is the observed sensitivity. The model includes photon noise from the sky and telescope based on the measured instrument transmission (25\%) and aperture efficiency of the telescope $(\sim 70 \%)$. The left and right panels show observation under atmosphere optical depths of $\tau_{225}=0.068$ and $\tau_{225}=0.171$, respectively. 
converts the $1 / \mathrm{Hz}^{1 / 2}$ to $\mathrm{sec}^{1 / 2}$ because one second of integration time corresponds to $0.5 \mathrm{~Hz}$ of bandwidth. The aperture efficiency of the CSO is about $75 \%$ at our frequency, and the net result is that we predict a flux sensitivity of about 0.4 Jy $\sqrt{\mathrm{sec}}$.

This fundamental noise sources allow us to make a sensitivity model mentioned above, and then, we compare the model with observed sensitivity (see section 2.1) in Figure 4. It shows that Z-Spec is now operating very close to the modeled sensitivity, which is dominated by irreducible photon noise from the sky and telescope. The left panel is the sensitivity at $\tau_{225} \leq 0.1$ and right panel is at $\tau_{225}>0.1$. It is obvious that the $\tau_{225} \leq 0.1$ sensitivity is lower than $\tau_{225}>0.1$ one. The important result revealed in the figure is that Z-Spec performance is very close to our theoretical sensitivity model and the method of the noise evaluations is valid. This means Z-Spec performance is now well understood and we can continue the long-lasting observations required for the faint high redshift submillimeter galaxies with nearly background limited performance.

\section{STATUS OF OBSERVATIONS OF NEARBY ULIRGS}

The $1 \mathrm{~mm}$ atmospheric window is rich with molecular rotational transitions which are very useful for investigation the physical and chemical conditions on the active central regions of LIRGs and ULIRGs without suffering from dust extinction. Unlike the previous spectroscopic study with heterodyne receivers, the broad spectral bandwidth of Z-Spec enables us to obtain strength of these molecular lines simultaneously. Notable tracers available in Z-Spec's bandpass are: $\mathrm{CO}$ isotopes $\mathrm{J}=2 \rightarrow 1$ (optically thick ${ }^{12} \mathrm{CO}$ for temperatures, optically thin ${ }^{13} \mathrm{CO}$ and $\mathrm{C}^{18} \mathrm{O}$ for mass), $\mathrm{HCN} \mathrm{J}=3 \rightarrow 2$ and $\mathrm{HNC} \mathrm{J}=3 \rightarrow 2$ (dense gas tracers), and $\mathrm{CN} \mathrm{J}=2 \rightarrow 1$ (a tracer for dense photodissociation regions around young stars). Z-Spec spectra will complement existing data from lower-J transitions of the same species previously obtained at lower frequencies. Recently, it has been suggested that millimeter-wave spectroscopic line ratios can be used as a diagnostic tool of the dominant energy source ${ }^{[17,18]}$. In particular, the $\mathrm{HNC} / \mathrm{HCN}$ ratio, the ratio of the $\mathrm{J}=1 \rightarrow 0 \mathrm{HCN}$ to $\mathrm{CO}$, and $\mathrm{HCO}^{+} / \mathrm{HCN}$ ratio are significantly different between galaxies dominated by AGN and starbursts.

After engineering runs at CSO in 2006, we have started observations of M82, LIRGs, and ULIRGs. Since M82 is enough to be spatially resolved by the $\sim 30$ arcsec beam of Z-Spec at CSO, spatial variation of the physical condition of the dense molecular gas can be also discussed ${ }^{[5]}$. At the moment more than dozen LIRGs and ULIRGs have been observed. It is notable that the spectrum of Arp 220 has relatively strong $\mathrm{HCN}, \mathrm{HNC}\left(\sim 20 \%\right.$ of $\left.{ }^{12} \mathrm{CO} 2 \rightarrow 1\right)$ emission lines which indicate the existence of warm and dense molecular gas, while these lines emitted from NGC 6240 are relatively weak indicating weaker contribution from the starburst. Moreover, in Arp 220 spectrum we note the first detection of $\mathrm{CCH} \mathrm{J}=3 \rightarrow 2$, a potential progenitor of the Polycyclic Aromatic Hydrocarbon molecules.

The typical integration time of observations of LIRGs and ULIRGs is several hours, and hence for weaker lines we could not reach to signal-to-noise ratio of five. We will therefore continue observations of such sources in the coming semesters.

\section{CONCLUSION}

We report the Z-Spec noise performance during observations at CSO and the compare them with a model of sensitivity. The Z-Spec observed sensitivity is estimated from the white noise level of the PSD of the bolometer output voltage. The model includes photon noise from the sky and telescope, based on the measured instrument transmission $(25 \%)$ and aperture efficiency of the telescope $(\sim 70 \%)$. We found the observed sensitivity is quite close to the model and performs as we expect.

The spectra of nearby ULIRGs are obtained with several molecular rotational lines. These lines are useful to explore the galaxies' chemical and physical conditions, such as gas mass and temperature, and some of these potentially can be used to diagnose AGN or starbursts which provide the energy for the huge luminosity of these galaxies. 


\section{ACKNOWLEGEMENTS}

We thank all the CSO staff for supporting us to perform the Z-Spec observations successfully. H. Inami thanks the graduate university of advanced study for supporting the travel cost for visiting Caltech and JPL to complete this work. L. Earle is supported by NASA GSRP fellowship (NGTS-50478). J. Aguirre is funded by a Jansky Fellowship from NRAO. J. Glenn acknowledges an NSF Career Grant in support of Z-Spec (AST-0239270) and an Innovation Award from the Research Corporation (RI0928). This work was supported in part by NASA SARA grants NAGS-11911 and NAGS-12788.

\section{REFERENCES}

[1] Bradford, C. M. et al. 2002, Proc. SPIE, 4850, 1137

[2] Naylor, B. J. et al. 2002, Proc. SPIE, 4855, 239

[3] Earle, L. et al. 2006, Proc. SPIE, 6275, 32

[4] Bradford, C. M. et al. 2004, Proc. SPIE, 5498, 257

[5] Naylor, B. J. 2007, Ph.D. Thesis, The California Institute of Technology

[6] Earle, L. et al. 2008, Proc. SPIE, 7020-39

[7] Rowland H. A., 1883, Philos. Mag., 16, 197

[8] Bradford, C. M. 2008, Proc. SPIE, 7020-61

[9] Holland, W. S. et al. 1999, $A \& A, 303,659$

[10] Bertoldi, F. et al. 2000, $A \& A, 360,92$

[11] Laurent, G. et al. 2005, $A p J, 623,742$

[12] Chapman, S.C. et al. 2005, $A p J, 622,772$

[13] Wang, W. H., Cowie, L. L. \& Barger, A. J. 2006, ApJ, 647, 74

[14] Scoville et al. 1991, ApJ, 366, L5

[15] Imanishi, M., Dudley, C. C., \& Maloney, P. R. 2006, ApJ, 637, 114

[16] Maloney, P. R. 1999, Ap\&SS, 266, 207

[17] Kohno, K. et al. 2001, ASP, 672

[18] Krips, M. et al. 2008, ApJ, 677, 275

[19] Zang, N.F. 2006, Metrologia, 43, 195 